\title{
Asociación entre motivación y rendimiento académico en los estudiantes del 1er ciclo de la carrera de Administración del Instituto de Educación Superior Tecnológico Privado Amazónico
}

Mg. Cinthya Torres Silva ctorresv@unsm.edu.pe ORCID: 0000-0002-8543-6975

Mtro. Roger Ricardo Rengifo Amasifen rrengifoa@unsm.edu.pe ORCID: 0000-0003-0579-6928

Mg. Wilfredo Torres Reátegui wtorres@unsm.edu.pe ORCID: 0000-0001-8811-0703

Mtro. José Gabriel Seijas Díaz Jseijasd@unsm.edu.pe

\section{RESUMEN}

ORCID: 0000-0002-7542-2254

El presente estudio tiene como objetivo Determinar la asociación que existe entre motivación y rendimiento académico en los estudiantes del 1er ciclo de la carrera de administración del Instituto de Educación Superior Tecnológico Privado Amazónico, de la ciudad de Tarapoto, Provincia y Región San Martín en el año 2014 y corresponde a un diseño de tipo descriptivo correlacional porque busca recoger información con la finalidad de describirla y Correlacional pues se busca establecer la asociación existente entre las variables motivación y rendimiento académico. El muestreo es de tipo población muestral, lo que indica el total de la población es la muestra. Consta de 30 estudiantes del primer ciclo del Instituto de Educación Superior Tecnológico Privado Amazónico. Los instrumentos utilizados fueron la Escala de motivación y los registros de la institución para recoger la información de rendimiento académico. Se concluye que existe asociación entre motivación y rendimiento académico en los estudiantes del primer ciclo del Instituto de Educación Superior Tecnológico Privado Amazónico, Tarapoto - 2014, obteniendo un $\mathrm{X}^{2}$ calculado el valor de 10,98 y con un margen de error de 0.10 y con 6 grados de libertad, dando como $\mathrm{X}^{2}$ tabular de 10,64. En relación a la motivación de mayor frecuencia es la orientación hacia el dominio y en la variable rendimiento académico es el regular.

Palabras clave: motivación y rendimiento académico. 


\title{
Association between motivation and academic performance in the students of the 1st cycle of the Administration career of the Instituto de Educación Superior Tecnológico Privado Amazónico
}

\begin{abstract}
ABSTRAC
This study aims to determine the relationship between motivation and academic achievement in students of 1st cycle race management of Tarapoto, San Martin province and region in the year 2014 and corresponds to a descriptive correlational design that seeks to collect information for the purpose of describing and correlational as it seeks to establish the association between the variable's motivation and academic performance The sampling rate of the sample population, indicating the total sample population. It consists of 30 undergraduate students from the Technological Institute of Higher Education Private Amazon, the city of Tarapoto, San Martin province and region in 2014. The instruments used were the Scale of motivation and the records of the institution to collect information on academic performance, with reference to its design and development, the Likert scale for level of motivation, an instrument that was validated by expert judgment regarding and reliability through Cronbach's alpha.

It concludes that there is an association between motivation and academic performance in undergraduate students of Technological Institute of Higher Education Private Amazon, Tarapoto - 2014, obtaining a $\mathrm{X}^{2}$ value of 10.98 calculated with a margin of error of 0.10 and 6 degrees of freedom, giving 10.64 tabular $\mathrm{X}^{2}$. Regarding motivation is more often the domain orientation and the academic performance variable is regular.
\end{abstract}

Keywords: motivation and academic performance.

Artículo recibido: 15 enero2020 Aceptado para publicación: 18 febrero2020 Correspondencia: ctorresv@unsm.edu.pe Conflictos de Interés: Ninguna que declarar 


\section{INTRODUCCIÓN}

Con el propósito de fundamentar la presente investigación, a continuación, se presentan algunas investigaciones realizadas según las variables en estudio, tanto en el ámbito internacional y nacional.

\section{Antecedente}

Así encontramos en el ámbito Internacional a:

Maquilón Sánchez, Javier J.; Hernández, Fuensanta (2011). Influencia de la motivación en el rendimiento académico de los estudiantes de formación profesional. Universidad de Murcia. España. REIFOP, 14 (1), 81-100. (Enlace web: http//www.aufop.com

A nivel de resultados, concluimos que seis de cada diez estudiantes de los ciclos formativos emplean predominantemente enfoque superficial, lo cual repercute muy negativamente en sus resultados académicos. Los enfoques profundos y de alto rendimiento son los menos empleados. A priori los estudiantes superficiales son catalogados como "malos" estudiantes, con inadecuadas motivaciones y estrategias para superar las demandas que se les pueda realizar a lo largo de sus estudios, pero para poder hacer esta tajante afirmación deberíamos saber si el proceso de enseñanza y los contenidos que se abordan en las asignaturas no están condicionando que los estudiantes se adapten a las demandas empleando un enfoque superficial. Es decir, que el ser superficiales sea más una estrategia que una motivación, ya que es lo demandado por los profesores en sus clases.

\section{Batista Silva Aliocha; Gálvez Espinos Maydel; Hinojosa Cueto Iliana (2010).}

Bosquejo histórico sobre las principales teorías de la motivación y su influencia en el proceso de enseñanza-aprendizaje. Rev cubana Med Gen Integr v.26 n.2 Ciudad de La Habana - Cuba.

Concluye

Que la motivación es un elemento importante del comportamiento organizacional del proceso enseñanza aprendizaje, que permite canalizar el esfuerzo, la energía y la conducta en general del estudiantado, permitiéndoles sentirse mejor respecto a lo que hacen y estimulándolos a trabajar más para el logro de los objetivos que interesan a la organización. Así mismo, la evaluación del desempeño constituye una técnica de dirección imprescindible en el aspecto administrativo del proceso enseñanza aprendizaje. 
Mediante ella se pueden detectar problemas en el desenvolvimiento del trabajo del recurso humano. El desempeño del binomio profesor-alumno es la piedra angular para desarrollar con efectividad y éxito este proceso. Siempre es importante aportar al conocimiento general sobre las motivaciones y el proceso enseñanza aprendizaje, lo que puede contribuir a tener una asistencia médica más eficaz y una docencia más perfeccionada.

Quinatoa Arellano Lourdes América (2010). La motivación en el área de informática durante la función mediadora del docente en el desarrollo del aprendizaje significativo en los estudiantes del séptimo año de educación básica en la escuela "Gabriela Mistral” del Cantón Chillanes, Provincia Bolívar durante el período lectivo 2009 - 2010. Universidad Estatal Bolívar. Ecuador.

Concluye:

La falta de técnicas de motivación afecta el desarrollo del aprendizaje significativo en los estudiantes del séptimo año de educación básica de la escuela Gabriela Mistral.

La falta de motivación en los estudiantes ha hecho que las clases se conviertan en una tarea rutinaria que ha causado en los estudiantes un desinterés y una falta de concentración que conlleva a un aprendizaje significativo inadecuado.

Narváez Terán Gustavo Alejandro (2011) “La motivación intrínseca y su influencia en el aprendizaje del idioma inglés en los estudiantes del cuarto ciclo de la carrera de informática educativa de la facultad de ciencias de la educación sociales, filosóficas y humanísticas en la Universidad Estatal de Bolívar, durante el período octubre 2010 marzo 2012”. Universidad Técnica de Ambato. Ecuador.

Concluye:

Como consecuencia de las encuestas realizadas a estudiantes y docentes de la Carrera de Informática Educativa de la Universidad Estatal de Bolívar, se entiende que a pesar de que las aulas cuentan con equipos audiovisuales para mejorar el aprendizaje del idioma inglés, los estudiantes en muchas ocasiones manifiestan que no son explotados de una manera positiva y así ellos se sientan motivados al recibir las clases de Inglés, por otro lado ellos nos hablan de que no existen aulas propias destinadas para recibir la materia, por el contrario, utilizan aulas prestadas de otras facultades o escuelas. 
Los estudiantes sienten que cuando reciben las clases de inglés los profesores no utilizan estrategias innovadoras que le permitan sentirse motivados intrínsecamente al recibir clases.

La Universidad Estatal de Bolívar en el pensum académico de la Escuela de Informática Educativa, los estudiantes deben aprobar tres niveles o ciclos de inglés (compuestos por 6 meses cada uno), a partir del tercer ciclo. La mayor parte de los estudiantes manifiestan que estudian la materia por ser parte de currículo, y por ende por pasar de año, es decir, no existe un deseo de ellos por aprender otro idioma, y los profesores opinan de igual manera.

Nacional: Cancho y Wilard (2010)." Relación entre estilos de aprendizaje y rendimiento académico de los alumnos de 1ero y 2 do grado del nivel secundario de la I.E.P."L. Fibonacci" de la ciudad de Lima. La mencionada investigación descriptiva correlacional se ha realizado en una muestra de 20 alumnos utilizando el cuestionario y actas de evaluación. El autor formula las siguientes conclusiones:

Existe relación significativa entre estilos de aprendizaje y rendimiento académico de los alumnos de primer y segundo grado del nivel secundario de la I.E.P "Leonardo Fibonacci”.

El tipo de estilo de aprendizaje predominante de los alumnos de primer y segundo grado del nivel secundario de la I.E.P "Leonardo Fibonacci" es el "reflexivo", alcanzando el 60 $\%$ del total.

Los alumnos que presentaron el estilo de aprendizaje "reflexivo" (60\%) su promedio ponderado estuvo ubicados en los parámetros de 15 y 18 de calificación, consolidando este estilo de aprendizaje como el generador de un óptimo rendimiento académico.

Los alumnos que presentaron el estilo de aprendizaje "pragmático" (5\%) su promedio ponderado estuvo ubicados en los parámetros de 10 y 14 de calificación, consolidando este estilo de aprendizaje como el generador de un pésimo o bajo rendimiento académico. El 50 \% de las mujeres predomina el estilo de aprendizaje "reflexivo" y en el varón el estilo de aprendizaje también es el "reflexivo" con un $60 \%$ del total.

Rhor García Godos Eva Matilde (2012). Relación de los factores, Autoestima, Motivación, Puntaje de Ingreso en el Rendimiento Académico de los alumnos ingresantes 2010, a la Universidad Nacional de Tumbes. Universidad Nacional de San Marcos.

Concluye: 
Con lo concerniente a la variable motivación personal, los datos nos llevan a concluir que la motivación personal es un factor muy significativo para lograr mejores rendimientos académicos. Esto es observado en todas las escuelas académicos profesionales donde encontramos que existe una relación muy significativa de la motivación en el rendimiento académico.

En cuanto a la variable puntaje de ingreso en relación con el rendimiento académico, observamos que sólo en las escuelas académico profesionales de Derecho y Gestión en Hotelería y Turismo, existe una relación, más no así en las demás escuelas. Pero de modo general (es decir todas las escuelas académicas profesionales), observamos que si existe una relación del puntaje de ingreso en el rendimiento académico.

Estos datos nos están indicando que el puntaje de ingreso se relaciona de manera peculiar con el rendimiento académico de acuerdo al área de formación académico profesional. Aplicando la Prueba de Pearson "r", los datos obtenidos respecto a la variable motivación, podemos concluir que existe una relación lineal positiva altamente significativa; donde el coeficiente de determinación muestra que el $95 \%$ de la variable rendimiento académico es explicado por el modelo de regresión. Si deseamos mejorar el rendimiento académico, es de suma importancia mejorar los niveles de motivación intrínseca especialmente.

La variable puntaje de ingreso tiene un comportamiento diferente en relación al rendimiento académico, encontrando que no existe una relación lineal positiva significativa, siendo el coeficiente de determinación el 17\% de la variable rendimiento académico explicado por el puntaje de ingreso.

El bajo rendimiento académico de los alumnos ingresantes 2010 en la Universidad Nacional de Tumbes, supone a nivel institucional, una disminución de la calidad de los programas, ya que el número de alumnos retrasados dará lugar a masificación de las aulas, mayor tiempo de permanencia en la universidad y por tanto, a un proceso de enseñanzaaprendizaje deficitario.

\section{ESTRATEGIAS METODOLÓGICAS O MATERIALES Y MÉTODOS}

El diseño de investigación se pude definir como una estructura u organización esquematizada que adopta el investigador para relacionar y controlar las variables de estudio, (Hernández 2010).

Un aspecto importante, es definir con claridad y de modo específico la población objetivo de la investigación. Para ello se debe tener determinadas las características de los 
elementos que posibiliten identificar la pertenencia o no a la población objetivo. (Hernández 2006).

El universo total de la población es de 30 estudiantes del primer ciclo de la carrera de administración del Instituto de Educación Superior Tecnológico Privado Amazónico, del Distrito de Tarapoto, periodo 2014.

La investigación tuvo un tipo aplicada, de diseño no experimental descriptiva, correlacional, transversal, con una población conformada por 174 colaboradores, se aplicó como técnica la encuesta y el fichaje, siendo el instrumento un cuestionario, los datos cuantitativos fueron procesados y analizados por medios electrónicos, clasificados y sistematizados de acuerdo a las unidades de análisis correspondientes, respecto a sus variables, a través de Microsoft Excel y el programa estadístico SPSS

Los instrumentos pasaron un proceso de validación y confiabilidad en cuanto al contenido de los Ítems del cuestionario aplicado a la población objeto de estudio, los cuales fueron desarrollados bajo la fundamentación teórica de las variables investigadas, bajo este contexto, la validez de esta investigación se determinó a través del método estadístico.

\section{RESULTADOS Y DISCUSIÓN}

Tabla 01. Distribución de frecuencias y porcentajes de motivación de los estudiantes del ler ciclo de la carrera de administración del Instituto de Educación Superior Tecnológico Privado Amazónico, de la ciudad de Tarapoto, Provincia y Región San Martín en el año 2014.

\begin{tabular}{|l|l|l|}
\hline Motivacion & Frecuencia & Porcentaje \\
\hline Orientacion hacia el dominio & 14 & $46.67 \%$ \\
\hline Orientacion hacia el desempeño & 9 & $30.00 \%$ \\
\hline Orientacion hacia la evitacion & 7 & $23.33 \%$ \\
\hline Total & $\mathbf{3 0}$ & $\mathbf{1 0 0 . 0 0 \%}$ \\
\hline
\end{tabular}

Fuente: Resultados Escala de motivación en los estudiantes del ler ciclo de la carrera de administración del Instituto de Educación Superior Tecnológico Privado Amazónico, de la ciudad de Tarapoto, Provincia y Región San Martín en el año 2014

En la tabla $\mathbf{N}^{\mathbf{0}} 01$ se puede identificar que la orientación hacia el dominio, es el tipo que se encuentra con mayor frecuencia, y la orientación hacia la evitación aparece con menor frecuencia en los estudiantes del 1er ciclo de la carrera de administración del Instituto de Educación Superior Tecnológico Privado Amazónico, de la ciudad de Tarapoto, Provincia y Región San Martín 2014. 
De los 30 estudiantes del 1er ciclo de la carrera de administración del Instituto de Educación Superior Tecnológico Privado Amazónico, de la ciudad de Tarapoto, Provincia y Región San Martín en el año 2014, se obtuvo que el 46.67\% de los estudiantes presenta rendimiento regular, lo que indica que el estudiante está en el camino de lograr los aprendizajes previstos y requiere acompañamiento durante un tiempo razonable para lograrlo

Tabla 02. Distribución de frecuencias y porcentajes de rendimiento académico en los estudiantes del ler ciclo de la carrera de administración del Instituto de Educación Superior Tecnológico Privado Amazónico, de la ciudad de Tarapoto, Provincia y Región San Martín en el año 2014

\begin{tabular}{|l|l|l|}
\hline Rendimiento academico & Frecuencia & Porcentaje \\
\hline Muy bueno & 0 & $0.00 \%$ \\
\hline Bueno & 5 & $16.67 \%$ \\
\hline Regular & 14 & $46.67 \%$ \\
\hline Mal & 11 & $36.67 \%$ \\
\hline Total & $\mathbf{3 0}$ & $\mathbf{1 0 0 . 0 0 \%}$ \\
\hline
\end{tabular}

Tabla $\mathbf{N}^{\circ}$ 03. - Tabla de contingencia de motivación y rendimiento académico en los estudiantes del ler ciclo de la carrera de administración del Instituto de Educación Superior Tecnológico Privado Amazónico, de la ciudad de Tarapoto, Provincia y Región San Martín en el año 2014

\begin{tabular}{|c|c|c|c|c|}
\hline \multirow[b]{2}{*}{$\begin{array}{l}\text { Rendimiento } \\
\text { academico }\end{array}$} & \multicolumn{3}{|l|}{ Motivacion } & \multirow[b]{2}{*}{ Total } \\
\hline & \begin{tabular}{|lr}
\multicolumn{3}{l}{ ORIENTACION } \\
HACIA & EL \\
DOMINIO &
\end{tabular} & $\begin{array}{l}\text { ORIENTACION } \\
\text { HACIA EL } \\
\text { DESEMPENOO }\end{array}$ & \begin{tabular}{|l} 
ORIENTACION \\
HACIA LA \\
EVITACION
\end{tabular} & \\
\hline Muy bueno & 0 & 0 & 0 & 0 \\
\hline Bueno & 1 & 2 & 2 & 5 \\
\hline Regular & 11 & 2 & 1 & 14 \\
\hline Mal & 2 & 5 & 4 & 11 \\
\hline Total & 14 & 9 & 7 & 30 \\
\hline
\end{tabular}

Fuente: Resultados Escala de motivacion y rendimiento academico en estudiantes del ler ciclo de la carrera de administración del Instituto de Educación Superior Tecnológico Privado Amazónico, de la ciudad de Tarapoto, Provincia y Región San Martín en el año 2014.

Tabla $\mathbf{N}^{\circ}$ 04. - Tabla de contingencia con puntajes esperados de motivación y rendimiento académico en los estudiantes del 1er ciclo de la carrera de administración 
del Instituto de Educación Superior Tecnológico Privado Amazónico, de la ciudad de

Tarapoto, Provincia y Región San Martín en el año 2014

\begin{tabular}{|c|c|c|c|c|}
\hline \multirow{3}{*}{$\begin{array}{l}\text { Rendimiento } \\
\text { academico }\end{array}$} & \multicolumn{3}{|l|}{ Motivacion } & \multirow{3}{*}{ Total } \\
\hline & ORIENTACION & ORIENTACION & ORIENTACION & \\
\hline & $\begin{array}{ll}\text { HACIA } & \text { EL } \\
\text { DOMINIO } & \end{array}$ & $\begin{array}{l}\text { HACIA EL } \\
\text { DESEMPEÑO }\end{array}$ & $\begin{array}{l}\text { HACIA LA } \\
\text { EVITACION }\end{array}$ & \\
\hline Muy bueno & $0(0.00)$ & $0(0.00)$ & $0(0.00)$ & 0 \\
\hline Bueno & $1(2.33)$ & $2(1.50)$ & $2(1.17)$ & 5 \\
\hline Regular & $11(6.53)$ & $2(4.20)$ & $1(3.27)$ & 14 \\
\hline Mal & $2(5.13)$ & $5(3.30)$ & $4(2,57)$ & 11 \\
\hline Total & 14 & 9 & 7 & 30 \\
\hline
\end{tabular}

Fuente: Resultados Escala de motivacion y rendimiento academico en estudiantes del ler ciclo de la carrera de administración del Instituto de Educación Superior Tecnológico Privado Amazónico, de la ciudad de Tarapoto, Provincia y Región San Martín en el año 2014.

Tabla $\mathbf{N}^{\circ}$ 05. - Comparación y decisión entre $X^{2} c$ y $X^{2} t$

\begin{tabular}{|l|l|l|l|l|l|}
\hline $\begin{array}{l}\text { Motivación y } \\
\text { rendimiento } \\
\text { académico }\end{array}$ & $\begin{array}{l}\text { Grados de } \\
\text { Libertad }\end{array}$ & $X^{2} \mathrm{c}$ & $\begin{array}{l}\text { Nivel de } \\
\text { significancia }\end{array}$ & $\mathrm{X}^{2} \mathrm{t}$ & Decisión \\
\cline { 2 - 6 } & 6 & 10.89 & 0.10 & 10,64 & Se rechaza la Ho \\
\hline
\end{tabular}

\begin{tabular}{|c|c|c|c|c|c|}
\hline \multicolumn{6}{|c|}{ Tabla 4. Distribución de ji-cuadrado } \\
\hline$P^{P(}$ & $\frac{\partial x}{x}$ & & & & \\
\hline $\begin{array}{ll}1 \\
0\end{array}$ & Probal & idad de & valor & erior & \\
\hline Grados de libertad & 0,1 & 0,05 & 0,025 & 0,01 & 0,005 \\
\hline 1 & 2,71 & 3,84 & 5,02 & 6,63 & 7,88 \\
\hline 2 & 4,61 & 5,99 & 7,38 & 9,21 & 10,60 \\
\hline 3 & 6,25 & 7,81 & 9,35 & 11,34 & 12,84 \\
\hline 4 & 7,78 & 9,49 & 11,14 & 13,28 & 14,86 \\
\hline 5 & 9,24 & 11,07 & 12,83 & 15,09 & 16,75 \\
\hline 6 & 10,64 & 12,59 & 14,45 & 16,81 & 18,55 \\
\hline 7 & 12,02 & 14,07 & 16,01 & 18,48 & 20,28 \\
\hline 8 & 13,36 & 15,51 & 17,53 & 20,09 & 21,95 \\
\hline
\end{tabular}


El estadístico $X^{2}$ c encontrado es 10.89 y con un nivel de confiabilidad del 90\% $(\alpha=0.10)$ el tabular es 10,64, lo que indica que ambas variables no son independientes. Por lo tanto, ambas están asociadas, eso permite concluir que, si se trabaja uno de ellas, en este caso la motivación, podemos mejorar el rendimiento académico en los estudiantes del 1er ciclo de la carrera de administración del Instituto de Educación Superior Tecnológico Privado Amazónico, de la ciudad de Tarapoto, Provincia y Región San Martín en el año 2014.

\section{Discusión}

La palabra "motivación", ha sufrido un cambio progresivo, desde el punto de vista conductista, hasta las orientaciones cognitivas actuales. Su polémica gira en torno a los factores que pueden influir y la diferencia entre el interés que se presenta por una tarea. Efectivamente, es obvio que las atribuciones del éxito o fracaso, son factores que determinan la motivación de los educandos. Sin embargo, su estudio es complicado, porque tiene a su disposición, muchas teorías para analizar, e investigaciones y tesis controversiales sobre el tema.

Es un hecho que la motivación influye en el aprendizaje, hasta el punto de llegar a ser uno de los principales objetivos de los profesores: motivar a sus estudiantes. Sin embargo, la falta de motivación es una de las causas importantes que se debe valorar en el fracaso de los estudiantes, especialmente cuando se fundamenta en la distancia establecida entre los actuantes del proceso. Así, se puede observar que la relación profesor estudiante, en el contexto universitario, se presenta, generalmente, en forma lejana, y la impersonalidad que se concibe no permite centralizar la atención en el sujeto que aprende, sino más bien la formación universitaria tiende a identificar el punto fundamental del aprendizaje en el sistema, entre el profesor y el contenido. Este modelo se introduce en una sociedad, cuya finalidad inmersa en un proceso globalizante, se ubica frente a intereses de carácter cuantitativo y sistemas técnicos de manejo de información, lo que desdeña el sistema de transmisión de conocimientos de forma humanizante, ya que deja de lado al sujeto que aprende.

El resultado obtenido nos muestra que existe asociación entre motivación y rendimiento académico de los estudiantes 1er ciclo de la carrera de administración del Instituto de Educación Superior Tecnológico Privado Amazónico, de la ciudad de Tarapoto, Provincia y Región San Martín en el año 2014. Sin embargo, Rhor García Godos Eva Matilde (2012). Concluye que la motivación personal es un factor muy significativo para 
lograr mejores rendimientos académicos. Esto es observado en todas las escuelas académicos profesionales donde existe una relación muy significativa de la motivación en el rendimiento académico. Aplicando la Prueba de Pearson " $r$ ", los datos obtenidos respecto a la variable motivación, concluye que existe una relación lineal positiva altamente significativa; donde el coeficiente de determinación muestra que el $95 \%$ del rendimiento académico es explicado por el modelo de regresión. Si deseamos mejorar el rendimiento académico, es de suma importancia mejorar los niveles de motivación intrínseca especialmente. A su vez, Maquilón, Javier; Hernández, Fuensanta (2011). A nivel de resultados, concluimos que seis de cada diez estudiantes de los ciclos formativos emplean predominantemente enfoque superficial, lo cual repercute muy negativamente en sus resultados académicos. Los enfoques profundos y de alto rendimiento son los menos empleados. A priori los estudiantes superficiales son catalogados como "malos" estudiantes, con inadecuadas motivaciones y estrategias para superar las demandas que se les pueda realizar a lo largo de sus estudios, pero para poder hacer esta tajante afirmación deberíamos saber si el proceso de enseñanza y los contenidos que se abordan en las asignaturas no están condicionando que los estudiantes se adapten a las demandas empleando un enfoque superficial. Es decir, que el ser superficiales sea más una estrategia que una motivación, ya que es lo demandado por los profesores en sus clases.

En relación a la variable motivación, presenta la motivación de orientación hacia el dominio en un 46.67\%. Esto difiere de Gustavo Alejandro Narváez Terán (2011) Como consecuencia de las encuestas realizadas a estudiantes y docentes de la Carrera de Informática Educativa de la Universidad Estatal de Bolívar, se entiende que a pesar de que las aulas cuentan con equipos audiovisuales para mejorar el aprendizaje del idioma inglés, los estudiantes en muchas ocasiones manifiestan que no son explotados de una manera positiva y así ellos se sientan motivados al recibir las clases de Inglés, por otro lado ellos nos hablan de que no existen aulas propias destinadas para recibir la materia, por el contrario, utilizan aulas prestadas de otras facultades o escuelas.. Por su parte, Quinatoa Arellano Lourdes América (2010). La falta de técnicas de motivación afecta el desarrollo del aprendizaje significativo en los estudiantes del séptimo año de educación básica de la escuela Gabriela Mistral. La falta de motivación en los estudiantes ha hecho que las clases se conviertan en una tarea rutinaria que ha causado en los estudiantes un desinterés y una falta de concentración que conlleva a un aprendizaje significativo 
inadecuado. En ese sentido, Batista, Aliocha; Gálvez, Maydel; Hinojosa, Iliana (2010). Concluye que la motivación es un elemento importante del comportamiento organizacional del proceso enseñanza aprendizaje, que permite canalizar el esfuerzo, la energía y la conducta en general del estudiantado, permitiéndoles sentirse mejor respecto a lo que hacen y estimulándolos a trabajar más para el logro de los objetivos que interesan a la organización. Así mismo, la evaluación del desempeño constituye una técnica de dirección imprescindible en el aspecto administrativo del proceso enseñanza aprendizaje. Mediante ella se pueden detectar problemas en el desenvolvimiento del trabajo del recurso humano.

En relación a la variable rendimiento académico, podemos indicar que el 53,57\% presentan un tipo de rendimiento regular. Esto presenta algunas coincidencias con Rhor García Godos Eva Matilde (2012). El bajo rendimiento académico de los alumnos ingresantes 2010 en la Universidad Nacional de Tumbes, supone a nivel institucional, una disminución de la calidad de los programas, ya que el número de alumnos retrasados dará lugar a masificación de las aulas, mayor tiempo de permanencia en la universidad y por tanto, a un proceso de enseñanza- aprendizaje deficitario.

\section{CONCLUSIÓN O CONSIDERACIONES FINALES}

\section{Conclusiones}

Se concluye que existe asociación entre motivación y rendimiento académico en los estudiantes del 1er ciclo de la carrera de administración del Instituto de Educación Superior Tecnológico Privado Amazónico, de la ciudad de Tarapoto, Provincia y Región San Martín en el año 2014, obteniendo $\mathrm{X}^{2=} 10.89$ y con un margen de error de 0.10 y con 06 grados de libertad $\mathrm{X}^{2}$ es 10.64; lo que indica que existe correlación entre ambas variables.

Los estudiantes del 1er ciclo de la carrera de administración del Instituto de Educación Superior Tecnológico Privado Amazónico, de la ciudad de Tarapoto, Provincia y Región San Martín en el año 2014, presenta el $46.67 \%$ motivación orientada hacia el dominio, esta se relaciona con ser la guía de la conducta de logro y el compromiso con la tarea. Los estudiantes con este tipo de orientación tienen el objetivo de aprender, comprender la información, desarrollar sus habilidades y lograr algo difícil de conseguir. Se relaciona positivamente con la autoeficacia, persistencia, preferencia por los retos, el aprendizaje 
autorregulado, el afecto positivo, autoestima, el énfasis en el aprendizaje y con la variedad de la tarea - incluye brindar diferentes tareas y trabajar.

Los estudiantes del 1er ciclo de la carrera de administración del Instituto de Educación Superior Tecnológico Privado Amazónico, de la ciudad de Tarapoto, Provincia y Región San Martín en el año 2014, presentan el 46.67\% rendimiento regular, lo que indica que el estudiante está en el camino de lograr los aprendizajes previstos y requiere acompañamiento durante un tiempo razonable para lograrlo., vergüenza, culpa, odio, tristeza, miedo y ansiedad.

\section{Recomendaciones}

Desarrollar un programa que incluya aspectos relacionados con la motivación en estudiantes del 1er ciclo de la carrera de administración del Instituto de Educación Superior Tecnológico Privado Amazónico, de la ciudad de Tarapoto, Provincia y Región San Martín en el año 2014. De esta manera resaltar su iniciativa y capacidad para poder mejorar sustancialmente ante situaciones de aprendizaje.

Promover comportamientos y conductas relacionados con el dominio y desempeño en estudiantes del 1er ciclo de la carrera de administración del Instituto de Educación Superior Tecnológico Privado Amazónico, de la ciudad de Tarapoto, Provincia y Región San Martín en el año 2014, que le permita a los adolescentes y jóvenes una mejor condición con el aprendizaje y promover una cultura de mérito y calidad de aprendizaje. Consolidar comportamientos y actitudes relacionadas con el aprendizaje y el rendimiento en estudiantes del 1er ciclo de la carrera de administración del Instituto de Educación Superior Tecnológico Privado Amazónico, de la ciudad de Tarapoto, Provincia y Región San Martín en el año 2014, que les permitan a las adolescentes y jóvenes mejorar sus capacidades de aprendizaje.

\section{LISTA DE REFERENCIAS}

Atkinson, J. W. (1957). Motivational determinants of risk-taking behavior, Psychological Review

Batista, Aliocha; Gálvez Espinos; Hinojos, Iliana (2010). Bosquejo histórico sobre las principales teorías de la motivación y su influencia en el proceso de enseñanzaaprendizaje. Rev cubana Med Gen Integr v.26 n.2 Ciudad de La Habana - Cuba. 
Bolaños, A. y Castro, M. (2001). El concepto de amistad que tienen los niños y niñas de dos jardines infantiles en la provincia de Heredia. (Tesis de Licenciatura no publicada). Universidad Nacional, Heredia, Costa Rica.

Campos, O. (2001). En la educación básica ¿valores o virtudes? Revista Iberoamericana de Educación, 1-5. Recuperado de http://www.rieoei.org/deloslectores/Campos.PDF

Cancho y Wilard (2010).” Relación entre estilos de aprendizaje y rendimiento académico de los alumnos de 1ero y 2 do grado del nivel secundario de la I.E.P."L. Fibonacci" de la ciudad de Lima.

CEA, M. (1999). Metodología cuantitativa. Estrategias y técnicas de investigación social. Madrid: Editorial Síntesis.

Cotterell, J. (1986). The Adjustment of Early Adolescent Youngsters to Secondary School: Some

De La Fuente, J., Peralta, F. y Sánchez, M. (2006). Valores sociopersonales y problemas de convivencia en la educación secundaria. Revista Electrónica de Investigación Psicoeducativa 4(2), 171-200.

Dessler, Gary (2001): Administración de personal, ( $8^{\circ}$ ed.), Pearson Prentice Hall Hispanoamericana., México, D.F.

Gimeno, J. (1997). La transición a la educación secundaria. Madrid, España: Ediciones Morata.

Graham, C. y Hill, M. (2003). Negotiating the transition to secondary school [La negociación en la transición a la secundaria]. University of Glasgow Edimburgo: Centro SCRE de la Universidad de Glasgow. Recuperado de http://www.preonline.co.uk/feature_pdfs/spot-light89.pdf

Hernández, R., Fernández, C., Baptista, P. (2003). Metodología de la investigación. México, D.F. McGraw-Hill Interamericana.

Herzberg F., B. Mauser and B. Snyderman, the Motivation to Work, Wiley, New York, 1959.

Hodgetts, R y Altman, S (1994). Comportamiento en las Organizaciones. Editorial McGraw-Hill. México.

Huertas, J. A. (1997). Motivación, querer aprender. Aique Grupo Editor S.A. Argentina. Kirkpatrick, D. (1997). Making the Change: Students' Experiences of the Transition to Primary School [Haciendo el cambio: experiencias de los estudiantes en la 
transición a la escuela primaria]. Recuperado de http://edoz.com.au/educationaustralia/archive/features/make.html

Maquilón Sánchez, Javier J.; Hernández Pina Fuensanta (2011). Influencia de la motivación en el rendimiento académico de los estudiantes de formación profesional. Universidad de Murcia. España. REIFOP, 14 (1), 81-100. (Enlace web: http//www.aufop.com).

McClellan, D. (1989). La motivación del logro, Estudio de la motivación humana. Editorial NARCEA, S. A. Madrid.

Meece, J., Anderman, E. \& Anderman, L. (2006). Classroom Goal Structure, Student Motivation and Academic Achievement. Annual Review Psychology.

Narváez, Alejandro (2011) “La motivación intrínseca y su influencia en el aprendizaje del idioma inglés en los estudiantes del cuarto ciclo de la carrera de informática educativa de la facultad de ciencias de la educación sociales, filosóficas y humanísticas en la Universidad Estatal de Bolívar, durante el período octubre 2010 - marzo 20112”. Universidad Técnica de Ambato. Ecuador.

Quinatoa, Arellano Lourdes (2010). La motivación en el área de informática durante la función mediadora del docente en el desarrollo del aprendizaje significativo en los estudiantes del séptimo año de educación básica en la escuela "Gabriela Mistral" del Cantón Chillanes, Provincia Bolívar durante el período lectivo 2009 - 2010. Universidad Estatal Bolívar. Ecuador.

Rhor, Godos (2012). Relación de los factores, Autoestima, Motivación, Puntaje de Ingreso en el Rendimiento Académico de los alumnos ingresantes 2010, a la Universidad Nacional de Tumbes. Universidad Nacional de San Marcos. 